\title{
III. Die DJV, die Neuorganisation des Gerichtssystems und die personelle „Säuberung“ der Justiz (1945-1947)
}

Am 4. September 1945 erließ der Oberste Chef der SMAD den für die Justizpolitik grundlegenden Befehl Nr. 49. Dieser dekretierte zum einen die Reorganisation des Gerichtswesens in der sowjetischen Zone in Übereinstimmung mit den Bestimmungen der vor dem 1. Januar 1933 gültigen Fassung des deutschen Gerichtsverfassungsgesetzes und griff ausdrücklich auf den traditionellen deutschen $\mathrm{Ge}$ richtsaufbau zurück. Zum anderen ordnete er an, daß gleichzeitig „aus dem Apparat der Gerichte und der Staatsanwaltschaft sämtliche früheren Mitglieder der NSDAP zu entfernen [seien], ebenso die Personen, welche unmittelbar teilgenommen haben an der Strafpolitik unter dem Hitler-Regime“. Die Durchführung des Befehls wurde dem Leiter der DJV, die Kontrolle darüber dem Chef der SMAD-Rechtsabteilung übertragen 1 .

Beim Erlaß von Befehl Nr. 49 dürften ein deutschlandpolitisches und ein pragmatisch-taktisches Motiv ausschlaggebend gewesen sein. Es ging der sowjetischen Seite einerseits darum, das für Gesamtdeutschland in den Verhandlungen im Alliierten Kontrollrat noch festzulegende Verfahren für die Reorganisation des deutschen Justizwesens zu präjudizieren². Dabei konnte die rigide Entnazifizierungsvorschrift des Befehls als konsequente Ausführung eines wesentlichen Beschlusses der Potsdamer Konferenz dargestellt werden, wenngleich dieser nur die Entfernung von NSDAP-Mitgliedern, "welche mehr als nominell an ihrer Tätigkeit teilgenommen haben "3, aus öffentlichen und halböffentlichen Ämtern und wichtigen Wirtschaftsunternehmen vorsah. In den Beratungen des Kontrollrats über das deutsche Gerichtswesen, die am 30. Oktober schließlich in Kontrollratsgesetz Nr. 4 mündeten, konnten sich die sowjetischen Vertreter jedoch nur teilweise durchsetzen. Es gelang ihnen zwar, entgegen den Vorstellungen der Briten und Amerikaner, die den Stand der Gerichtsverfassung des Jahres 1944 zugrunde legen wollten ${ }^{4}$, die Verhältnisse vor 1933 festzuschreiben; mit der Beschränkung auf die NSDAP-Mitglieder unter den Richtern und Staatsanwälten, „die sich aktiv für deren Tätigkeit eingesetzt haben“, sowie auf „alle anderen Personen, die an den Strafmethoden des Hitlerregimes direkten Anteil hatten“, war jedoch die Entfer-

1 Gedruckt in: Um ein antifaschistisch-demokratisches Deutschland, S. 142 f.

2 Vgl. u.a. Welsh, Revolutionärer Wandel, S. 134.

3 Es handelt sich um Punkt 6 von Teil A der Potsdamer Erklärung vom 2. 8. 1945, in: Amtsblatt des Kontrollrats, Ergänzungsblatt Nr. 1, S. 15.

+ So Karassjow in einem Bericht an Wyschinski vom 4.11. 1945; seine Darstellung, nach der der Kontrollrat die sowjetischen Entwürfe übernommen habe, ist jedoch unzutreffend. Der Bericht wurde dem Verfasser von Dr. Jochen Laufer freundlicherweise zur Verfügung gestellt. 
nung eines sehr viel kleineren Personenkreises anvisiert, als ihn der SMAD-Befehl vorgesehen hatte ${ }^{5}$. Dabei ist zu bedenken, daß es innerhalb der SMAD auch Bestrebungen gab, die Entnazifizierung der Justiz noch rigoroser zu betreiben als dies Befehl Nr. 49 vorschrieb: Denn in einer vom sowjetischen Außenministerium bestätigten Verordnung zur Ernennung von Richtern und Staatsanwälten vom April 1946 wurde festgelegt, alle im Justizdienst des Dritten Reiches Beschäftigten nicht wieder einzustellen. Dies ging jedoch der Rechtsabteilung zu weit, so daß sie mit Zustimmung des Politischen Beraters der SMAD nicht nach dieser Regelung verfuhr6.

Im Hinblick auf die eigene Zone standen für die Sowjetunion das Bedürfnis, durch Anknüpfung an die Verhältnisse vor 1933 sich die „Bündnispartner“ der KPD aus dem bürgerlichen Lager zu sichern, sowie die Notwendigkeit, das Gerichtswesen und die Entnazifizierungspraxis in der Justiz zu vereinheitlichen, im Mittelpunkt ${ }^{7}$. Im Zuge der militärischen Besetzung waren auf Anordnung der lokalen Kommandanten die unterschiedlichsten Gerichte gebildet, Nationalsozialisten aus Justiz und Staatsanwaltschaft entfernt und für die Rechtsprechung oftmals unbelastete Juristen und Nicht-Juristen herangezogen worden ${ }^{8}$. Erst als innerhalb der Landes- und Provinzialverwaltungen im Juli 1945 auch Justizabteilungen entstanden, konnte, in Ausführung der Weisungen der vorgesetzten Militärverwaltungen, die Vereinheitlichung von Gerichtsorganisation und Entnazifizierungspraxis in Angriff genommen werden. Freilich bestanden weiterhin Unterschiede zwischen den einzelnen Ländern und Provinzen', und auch einige der errichteten „Sondergerichte“ erwiesen sich als ausgesprochen zählebig ${ }^{10}$.

Befehl Nr. 49 ist zudem mit seiner Anordnung einer umfassenden personellen „Säuberung“ des Justizwesens von ehemaligen Nationalsozialisten in die Entnazifizierungspolitik insgesamt einzuordnen. Da mit der Entnazifizierung der zentralen Sektoren Verwaltung, Schule und Justiz in der SBZ vor allem "die Machtbasis der vorher regierenden Gruppen "11 aufgebrochen werden sollte, ging die sowjetische Militärverwaltung äußerst radikal vor. Bei der Anweisung des stellvertretenden Chefs der SMA der Provinz Sachsen, „das Gericht und die Staatsanwaltschaft [...] schnellstens von allen Nazis" zu bereinigen ${ }^{12}$, handelte es sich daher wohl kaum um einen Einzelfall. Indem die Besatzungsmacht die Entnazifizierung des Justizpersonals jedoch nicht in der Kompetenz der Landes- und Provinzialverwaltungen beließ, sondern mittels Befehl Nr. 49 zentralisierte, demonstrierte sie

5 Gedruckt in: Amtsblatt des Kontrollrats, Nr. 2, 30. 11. 1945, S. $26 \mathrm{f}$.

6 Vgl. Nikitin, Sowjetische Militäradministration und die Justiz (Manuskript), S. 8.

7 Das zweite Motiv wird ausdrücklich am Anfang des Befehls genannt.

8 Vgl. u.a. Tätigkeitsbericht der Justizabteilung der Provinzialregierung Mark Brandenburg (Mitte 1945-5. 7. 1946), in: Berichte der Landes- und Provinzialverwaltungen, S. 292, sowie Anders, Demokratisierung, S. 8-13.

9 Vgl. den Bericht Karassjows an Wyschinski, 4.11. 1945 (wie Anm. 4).

to Zum Teil bis Januar 1946: siehe Chef der DJV an Landes- bzw. Provinzialverwaltungen/Abteilung Justiz, Januar 1946, Entwurf, BAB, DP1 VA Nr. 2, Bl. 184.

11 Krieger, Interregnum, S. 42.

12 Niederschrift über eine Beratung der Provinzialverwaltung Sachsen mit Generalmajor Alexandr G. Kotikow über aktuelle Aufgaben und Probleme vom 18. 8. 1945, in: Berichte der Landes- und Provinzialverwaltungen, S. 83. 
zusätzlich, welche Bedeutung sie der „Säuberung“ dieses Sektors beimaß13. Die DJV bekam damit erstmals eine Aufgabe von herausragender Bedeutung von der Besatzungsmacht zugewiesen. Die in der Zentralverwaltung 1945 maßgebende Auffassung Schiffers ließ keinen Zweifel daran, daß die „Säuberung“ des Justizapparats hier ebenfalls höchste Priorität besaß. Denn für den DJV-Präsidenten stand "die Frage der Ausrottung des Nazismus“ nicht nur in einem allgemeinen Sinne „im Vordergrund der aktuellen politischen Probleme“14. Zur Überwindung der Kluft zwischen Volk und Recht wollte er unter anderem das der Justiz entgegengebrachte Vertrauen wiederherstellen, das durch die verscherzt worden sei, „die durch die Zugehörigkeit zur [nationalsozialistischen] Partei oder durch ihre Beteiligung an nazistischer Rechtsbrechung ein Kainszeichen auf die Richterrobe geheftet" hätten. Die „Säuberung“ der Justiz mußte daher „eine tiefergreifende sein als sonst irgendwo", nicht weil man die NS-Juristen härter als andere Berufsgruppen bestrafen müsse, sondern weil nur so das Mißtrauen gegen die Justiz überwunden werden könne ${ }^{15}$. Auch wenn die Motive andere waren, verfolgten die SMAD und Schiffer in diesem Kontext ein ähnliches Ziel. Gleichzeitig wollte Schiffer das elementare sowjetische Interesse an der Durchführung von Befehl Nr. 49 zur Unterordnung der Landesjustizverwaltungen unter den Führungsanspruch der DJV nutzen ${ }^{16}$ : Wenngleich nicht zur Unterstützung seiner Arbeit erlassen ${ }^{17}$, sah Schiffer in der Beauftragung mit der Durchführung des Befehls die entscheidende Möglichkeit, um seine Verwaltung handlungsfähig zu machen.

Zur Umsetzung des Befehls, die Schiffer mit einer allgemeinen Bestandsaufnahme des Justizwesens zu verbinden gedachte, reisten am 21. September aus DJV-Vertretern zusammengesetzte Kommissionen zu den Landes- und Provinzialverwaltungen, um sich vor Ort insbesondere über Art, Anzahl und Besetzung der Gerichte zu informieren und die zur Durchführung des Befehls notwendigen Maßnahmen zu treffen ${ }^{18}$. Einen Tag zuvor hatte Schiffer in einem Erlaß alle Justizverwaltungen angewiesen, nicht nur die früheren NSDAP-Mitglieder, sondern auch die Angehörigen der NSDAP-Gliederungen aus dem Dienst zu entfernen ${ }^{19}$. Diese Erweiterung der von der Entnazifizierung betroffenen Personengruppen, die durch den Befehlstext nicht gedeckt war, nahm Schiffer wohl im Einvernehmen mit Karlshorst vor. Dies legt zum einen die Formulierung der offiziellen sowjetischen Bekanntmachung des Befehls nahe, derzufolge „die Gerichtsorgane

13 In dem von Meinicke, Entnazifizierung, entwickelten Vier-Phasen-Modell, das auch von der westdeutschen Historiographie übernommen wurde, ist die Entnazifizierung nach Befehl Nr. 49 der zweiten Phase (Juli 1945-Ende 1946) zuzuordnen, die primär durch die Säuberungen auf der Grundlage von Landesgesetzen und -verordnungen geprägt war. $\mathrm{Da}$ dieses Modell nicht auf alle Regionen der SBZ übertragbar ist, hat van Melis, Entnazifizierung in Mecklenburg-Vorpommern, S. $10 \mathrm{f}$, gezeigt.

14 Schiffer, 8. Mai, S. 2.

is Ders., Deutsche Justiz, 2. Aufl., S. 27.

16 Siehe Kap. A.II.1.

17 So irrtümlich Meinicke, Entnazifizierung, S. 142.

18 Vgl. Rundverfügung an die Justizabteilungen der Landes- und Provinzialverwaltungen, 18.9. 1945, und Vermerk Rosenthal-Pelldrams für Kleikamp, Bertz, Kuenzer und Lentz, o.D., BAB, DP1 VA Nr. 2, Bl. 25-27, 29 f.

19 Der Erlaß vom 20. 9. 1945 war nicht aufzufinden; zu seinem Inhalt Chef der DJV an Landesverwaltung Sachsen/Abteilung Justiz, 3. 10. 1945, ebenda, Bl. 50, sowie Bericht über die Durchführung des Befehls Nr. 49, 20. 10. 1945, BAB, DP1 VA Nr. 614, Bl. 19. 
und die Staatsanwaltschaften aus solchen Personen zusammenzusetzen [waren], die die Sicherung der Aufgaben zur Ausrottung des Nazismus und zur Festigung der Demokratie garantieren"20. Zum anderen bezog sich auch für Karassjow die Entnazifizierungsvorschrift von Befehl Nr. 49 nicht nur auf die Parteimitglieder, sondern auch auf die Angehörigen der NSDAP-Gliederungen ${ }^{21}$. Die Bedeutung, die die SMAD-Rechtsabteilung der Durchführung des Befehls beimaß, geht nicht zuletzt daraus hervor, daß sie zur Überwachung seiner Durchführung eigene Vertreter in die Länder und Provinzen entsandte ${ }^{22}$.

Im Hinblick auf die Gerichtsorganisation gab es kaum Differenzen zwischen den Kommissionen aus der DJV und den Verantwortlichen vor Ort. So akzeptierte die DJV die Vorschläge der sächsischen Landesverwaltung im Hinblick auf Anzahl und Lage der Gerichte ${ }^{23}$. In Brandenburg war durch die dortige sowjetische Militärverwaltung angeregt worden, die Landgerichtsbezirke "den geschaffenen Oberlandratsämtern anzupassen", so daß vier Landgerichte in Cottbus, Eberswalde, Neuruppin und Potsdam errichtet wurden. Präsident Steinhoff hatte sich anfänglich aus Kostengründen gegen ein brandenburgisches Oberlandesgericht ausgeprochen, stimmte aber schließlich dessen Errichtung ,in Potsdam auf sparsamster Grundlage zu“24. Auch in Mecklenburg kam es über die Festlegungen bezüglich der Gerichtsorganisation anscheinend nicht zu Auseinandersetzungen. Die Neuorganisation des Gerichtswesens in der Provinz Sachsen erwies sich am schwierigsten, da die SMA in Halle bereits eine Neuordnung nach dem Grundsatz eingeleitet hatte, „daß die Gerichtsbezirke sich mit den politischen Verwaltungsbezirken decken" sollten. Demzufolge waren dort neben dem Oberlandesgericht drei Bezirksgerichte, 17 Stadtgerichte und 32 Kreisgerichte vorgesehen. Auch hier wurde jedoch im Einvernehmen mit der Provinzialverwaltung die dreigliedrige Gerichtsstruktur mit sieben Landgerichten eingeführt. Die Kontroverse über den Sitz des Oberlandesgerichts, bei der sich die Vertreter der Parteien, insbesondere aber die Kommunisten für Halle, Präsident Hübener und der Leiter der DJVKommission, Lentz, für Naumburg erklärten, wurde Anfang Oktober zugunsten Naumburgs entschieden ${ }^{25}$. In Thüringen konnten sich die DJV-Kommission und die Landesverwaltung zwar über die Reduzierung der Landgerichte von neun auf sieben sowie über die Streichung von 22 Amtsgerichten einigen, nicht aber über den Sitz des Oberlandesgerichts. Landespräsident Paul und Oberlandesgerichtspräsident Arno Barth hatten im August 1945 dessen Verlegung von Jena nach

20 Befehle des Obersten Chefs der SMAD, S. 37.

21 Bericht Karassjows an Wyschinski, 4. 11.1945 (wie Anm. 4).

22 Ebenda. Nach Nikitin, Sowjetische Militäradministration und die Justiz (Manuskript), S. 7 f., wurden Nikolajew, Jeroma, Wassilkow, Paschkewitsch, Lyssjak und Kurbatow in die SBZ geschickt. Vgl. auch den Bericht über die Dienstreise in den Oberlandesgerichtsbezirk Naumburg vom 21. bis 27. 9. 1945, BAB, DP1 VA Nr. 2, Bl. 42, in dem Lentz die Zusammenkunft mit Kurbatow schildert.

23 Die folgenden Ausführungen beruhen, wenn nicht anders vermerkt, auf dem Bericht über die Durchführung des Befehls Nr. 49, 20. 10. 1945, BAB, DP1 VA Nr. 614, Bl. 4-26.

24 Vermerk Georg Remak, 26. 9. 1946, ebenda, Bl. 36.

25 Bericht über die Dienstreise in den Oberlandesgerichtsbezirk Naumburg vom 21. bis 27. 9. 1945, BAB, DP1 VA Nr. 2, Bl. 43 f.; Bericht über die Reise nach Halle, Leipzig und Dresden vom 4. bis 6. 10. 1945, ebenda, Bl. 59. Das Oberlandesgericht wurde bis Jahresende nach Naumburg verlegt (Lorenz, Zentralverwaltung, S. 155), ab März 1946 war es jedoch wieder in Halle (Auskunft vom HStA Magdeburg vom 30. 9. 1997). 
Gera veranlaßt; die DJV-Kommission beharrte indes auf Jena. Wenngleich Schiffer in seinem Bericht vom 20. Oktober ankündigte, er „werde unverzüglich die für die Rückverlegung erforderlichen Maßnahmen treffen“, konnte er sich nicht durchsetzen, so daß das Gericht in Gera blieb"26. Insgesamt jedoch war die Gerichtsorganisation ohne nennenswerte Auseinandersetzungen nach den Grundsätzen von Befehl Nr. 49 wieder aufgebaut worden. Dies hing zum einen damit zusammen, daß eine einheitliche Gerichtsstruktur auch im Interesse der Länder und Provinzen lag, und zum anderen mit der Unterstützung der Vereinheitlichungsbestrebungen durch die sowjetischen Militäradministrationen in den jeweiligen Hauptstädten. Die getroffenen Regelungen erwiesen sich als so stabil, daß die Gerichtsorganisation nach dem Stand vom 1. Juli 1947 nur geringfügig von den knapp zwei Jahre zurückliegenden Festlegungen abwich ${ }^{27}$.

Größere Probleme bereitete die nach Befehl Nr. 49 durchzuführende rigorose Entnazifizierung der Justiz. Denn in dem Gebiet der späteren SBZ hatten 1939 knapp 80 Prozent der Richter und Staatsanwälte der NSDAP angehört, wobei die Prozentzahlen je nach Region zwischen rund 70 und 90 differierten ${ }^{28}$. Als die DJV-Kommissionen Ende September an ihren Bestimmungsorten weilten, war bereits ein Großteil der ehemaligen Parteigenossen aus der Justiz entlassen worden, wenngleich das Ausmaß der Entnazifizierung in den einzelnen Ländern und Provinzen höchst unterschiedlich war ${ }^{29}$. Zunächst kam es lediglich im Land und in der Provinz Sachsen zu Auseinandersetzungen um die Entfernung der noch verbliebenen Nationalsozialisten. Der Leiter der sächsischen Justizabteilung, Reinhard Uhle, legte am 26. September in einer Konferenz mit DJV-Vertretern dar, daß bei der Entfernung der noch im Justizdienst befindlichen ehemaligen 89 NS-Richter und -Staatsanwälte „keine Gewähr mehr für eine ordnungsgemäße Abwicklung der Rechtspflege in Sachsen " bestünde. Er bat daher, bei der SMAD vorstellig zu werden und dort eine einstweilige Belassung dieser Kräfte zu erwirken, die er als „nominell[e]“, ja sogar "gegnerisch eingestellt[e]“ Parteimitglieder bezeichnete. Schiffer bat zwar um eine Verringerung der 89 „Belasteten“, sagte aber zu, sich bei der Besatzungsmacht um „eine vorläufige Weiterbelassung eines Teiles der Kräfte“ einzusetzen ${ }^{30}$. Nach Rücksprache bei der SMAD-Rechtsabteilung sah er sich indes nicht mehr in der Lage, dem Wunsch Uhles zu entsprechen, und wies ihn am 3. Oktober an, den DJV-Erlaß vom 20. September „ohne Ausnahme“ durchzuführen ${ }^{31}$. In der Provinz Sachsen ergab sich ein ähnliches Pro-

26 Zu dem Konflikt siehe u.a. den Bericht Rosenthal-Pelldrams über den Kommissionsaufenthalt in Thüringen vom 22. bis 25. 9. 1945 sowie Barth an Schiffer, 25. 9. 1945, Paul an DJV, 10. 10. 1945, und das (nicht abgesandte) Schreiben Schiffers an Paul, 18.10. 1945. Alle Dokumente sowie ein Gutachten von Rosenthal-Pelldram, Vössing und Büchs, o.D., in BAB, DP1 VA Nr. 6, Bl. 15-28, 120f., 127, 132, 45-49. Vgl. dazu auch Weber, Justiz und Diktatur, S. 34.

27 Vgl. Vössing, Gerichtsorganisation, S. $142 \mathrm{f}$.

28 Die genauen Zahlen in: Benjamin u. a., Zur Geschichte der Rechtspflege 1945-1949, S. 333, allerdings mit falschen Prozentzahlen; richtig u.a. in: Meinicke, Entnazifizierung, S. LXI, Lorenz, Zentralverwaltung, S. 157.

29 Vgl. die Aufstellung in: Benjamin u.a., Zur Geschichte der Rechtspflege 1945-1949, S. 333; diese geht freilich von der Gesamtzahl der in der Justiz Beschäftigten aus.

30 Auszüge aus einem Bericht über die Konferenz vom 26. 9. 1945 zwischen Mitgliedern der sächsischen Landesverwaltung und der Zentralen Justizverwaltung, SAPMO, NY 4174/6, Bl.94.

31 Chef der DJV an Landesverwaltung Sachsen/Abteilung Justiz, 3. 10. 1945, BAB, DP1 VA Nr. 2, Bl. 50 . 
blem. Gegenüber der DJV-Kommission sprachen sich Präsident Hübener und Oberlandesgerichtspräsident Walter Brockmann ebenfalls gegen die Entlassung der noch tätigen, aber überprüften 99 NS-Richter und -Staatsanwälte aus. Lentz vermerkte, daß die Blockparteien - mit Ausnahme der Kommunisten -, die Gewerkschaften sowie die örtliche Militärverwaltung keine Einwände dagegen erhoben, so daß auch er sich diesem Votum anschloß, zumal die sofortige Entfernung der „Belasteten“ „zu einer vollkommenen Lähmung der Rechtspflege“ geführt hätte. Die Beteiligung der sowjetischen Militärverwaltung der Provinz Sachsen an dem Prüfungsverfahren für die „belasteten“ Juristen war für ihn ausschlaggebend ${ }^{32}$. Auch in der Provinz Sachsen wollte die SMAD-Rechtsabteilung jedoch keine Ausnahmen zulassen, so daß Schiffer am 3. Oktober Hübener aufforderte, umgehend alle von Befehl Nr. 49 betroffenen Personen aus dem Justizdienst zu entlassen ${ }^{33}$. Diese Vorfälle verdeutlichen, daß die DJV-Spitze trotz grundsätzlicher Befürwortung der durch Befehl Nr. 49 vorgegebenen Linie bereit war, zeitlich begrenzte Ausnahmen von der strikten Entnazifizierung zuzulassen, um die Funktionsfähigkeit des Justizwesens zu erhalten ${ }^{34}$. In dieser für die Besatzungsmacht zentralen Angelegenheit hatten pragmatische Argumente jedoch keine Chance, so daß die DJV gezwungen war, die konsequente Umsetzung der sowjetischen Vorgaben zu fordern.

Während das Land Sachsen die Entnazifizierung des Justizpersonals aufgrund der DJV-Anweisung anscheinend umfassend durchführte ${ }^{35}$, blieb die Provinz Sachsen ein Problemfall. Aufgrund einer Anordnung der SMAD-Rechtsabteilung übergab Rosenthal-Pelldram persönlich Schiffers Weisung vom 3. Oktober in Halle, wo ihm deren sofortige Ausführung zugesichert wurde ${ }^{36}$. Von den „belasteten" Justizjuristen blieben jedoch - ohne Wissen Schiffers ${ }^{37}$ - zahlreiche im Dienst. Fast sieben Monate später gab Karassjow Schiffer zu verstehen, daß man auf sowjetischer Seite „den Befehl Nr. 49, sofern er sich auf die Entfernung der faschistischen Elemente aus der Justiz bezieht, nicht schon für ausgeführt" halte. Dabei verwies er auf eine ihm zugegangene Mitteilung des Leiters der provinzsächsischen Justizabteilung, Erich Heine, derzufolge auch weiterhin ehemalige Nationalsozialisten im dortigen Justizwesen tätig seien ${ }^{38}$. Bald darauf, am 15. Juni

32 Bericht über die Dienstreise in den Oberlandesgerichtsbezirk Naumburg vom 21. bis 27.9. 1945, 28. 9. 1945, ebenda, Bl. 40, $44 \mathrm{f}$.

33 Chef der DJV an Präsident der Provinz Sachsen, 3. 10. 1945, ebenda, Bl. 49.

34 Abwegig ist daher der Versuch von Anders, Demokratisierung, S. 42, zwischen dem inkonsequenten Verhalten von Lentz (und anderen bürgerlichen Kräften in der DJV) und der von Schiffer angeblich vertretenen unnachgiebigen Linie bei der Säuberung des Justizpersonals zu unterscheiden.

35 Bericht Benjamins über die Durchführung des Befehls Nr. 49, vorgetragen in Karlshorst am 18.11. 1946, BAB, DP1 VA Nr. 996, Bl. 47-50, hier 48.

36 Bericht über die Reise nach Halle, Leipzig und Dresden vom 4. bis 6.10. 1945, BAB, DP1 VA Nr. 2, Bl. 57.

37 Schiffer berichtete am 20.10. 1945 der SMAD-Rechtsabteilung, sein Erlaß sei "unverzüglich“ durchgeführt worden, BAB, DP1 VA Nr. 614, Bl. 19. Lediglich im Fall des 1. Vizepräsidenten des Oberlandesgerichts, Friedrich Müller, NSDAP-Mitglied von 1933 bis 1934/35, der wegen Vergehens gegen das Heimtückegesetz 1939 zu einer vierjährigen Freiheitsstrafe verurteilt worden sei und nach Verbüßung seiner Strafe bis zum April 1945 im Konzentrationslager Buchenwald eingesessen habe, habe er dem Wunsch der Provinzialregierung, ihn über den 1.10. 1945 hinaus im Dienst zu belassen, Rechnung getragen.

38 Chef der SMAD-Rechtsabteilung an Chef der DJV, 18. 5. 1946, BAB, DP1 VA Nr. 2, Bl. 227-230, hier $228 \mathrm{f}$. 
1946, fand eine Unterredung zwischen Jakupow, Schiffer, Melsheimer und Heine statt. Auf die Aufforderung Jakupows, „schnellstens“ Befehl Nr. 49 umzusetzen, entgegnete Heine, daß von den 600 benötigten Richtern und Staatsanwälten in der Provinz nur 200 vorhanden seien. Unter diesen wiederum seien „57 noch nazistisch belastet“. Deren Entlassung würde die Funktionsfähigkeit der Justizbehörden in Frage stellen, da ein Ersatz nicht vorhanden sei und man nur auf Richter und Staatsanwälte ohne jegliche Vorbildung - von denen damals bereits 54 eingestellt waren ${ }^{39}$ - zurückgreifen könne. Zwar hielt auch Jakupow offensichtlich nicht viel von der Einsetzung dieser sogenannten „Richter im Soforteinsatz"40; dennoch änderte sich die sowjetische Linie nicht. Am 18. September 1946 wurde der Chef der DJV von Oberst Bukanow aufgefordert, die Entlassung der im Justizwesen der Provinz Sachsen noch tätigen 22 ehemals der NSDAP oder ihren Gliederungen angehörenden Personen zu verfügen ${ }^{41}$. Vor der SMAD-Rechtsabteilung konnte Hilde Benjamin am 18. November immerhin als Erfolg vermelden, $\mathrm{daß}$ von den am 28. Juni 1946 noch tätigen 56 NSDAP-Mitgliedern mittlerweile 48 entfernt seien ${ }^{42}$. Sie wußte freilich, daß die Provinzialregierung um den Verbleib der restlichen acht Richter kämpfte und gab DJV-intern zu, daß es nicht genüge, „die Richter zu beseitigen, man müsse auch Ersatzkräfte an ihrer Stelle heranholen" ${ }^{43}$. Obgleich sie bei derselben Gelegenheit die günstigen Erfahrungen mit juristisch nicht vorgebildeten Richtern betonte, war das Dilemma, vor das die rigide Entnazifizierung der Justiz die DJV stellte, deutlich geworden: Man konnte nicht ständig Entlassungen von qualifiziertem Personal anordnen, ohne die entstandenen Lücken mit gleichwertigem Ersatz aufzufüllen.

Auch über das Jahr 1946 hinaus hielten die Auseinandersetzungen mit SachsenAnhalt 44 an. Auf der Länderkonferenz vom 11. April 1947 sprach Jakupow die dortigen Mängel bei der Entnazifizierung der Justiz erneut an: Die meisten „nazistischen Elemente“ befänden sich zwar in der Anwaltschaft, jedoch seien auch in der Staatsanwaltschaft noch acht ehemalige Nationalsozialisten tätig. Darauf erwiderte Hübener, daß in Sachsen-Anhalt aufgrund des dramatischen Richtermangels ein Zusammenbruch der Justiz bereits erfolgt sei; in der freiwilligen Gerichtsbarkeit müßten daher auch "leicht belastete nominelle Pgs" beschäftigt werden, um die unbelasteten Juristen in der Staatsanwaltschaft und in den Spruchkammern einsetzen zu können ${ }^{45}$. Hübener beantragte daher am 19. April 1947 bei der Sowjetischen Militäradministration in Halle, „bis zu 50 wenig belastete und für den heutigen Aufbau tragbare nominelle Mitglieder der Nazipartei als juristische

39 Vermerk Winkelmann, 9. 7. 1946, BAB, DP1 SE Nr. 3474, B1. 3.

40 Aktenvermerk über eine Rücksprache mit Jakupow am 15.6. 1945, 17.6. 1946, BAB, DP1 SE Nr. 2221, Bl. 110.

41 SMAD-Rechtsabteilung an Chef der DJV, 18. 9. 1946, BAB, DP1 VA Nr. 7615.

42 Bericht über die Durchführung des Befehls Nr. 49, BAB, DP1 VA Nr. 996, Bl. 47-50, hier 49.

43 Vermerk über die Besprechung vom 16. 10. 1946, BAB, DP1 VA Nr. 7354.

44 Ende Dezember 1946 änderte der Landtag den Namen der ehemaligen preußischen Provinz in „Provinz Sachsen-Anhalt“; nach der Auflösung des preußischen Staates durch Kontrollratsgesetz Nr. 46 vom 25. 2. 1947 erhielt es durch SMAD-Befehl Nr. 180 vom 21. 7. 1947 und nach einem entsprechenden Landtagsbeschluß vom 27. 7. 1947 den Status eines "Landes Sachsen-Anhalt": vgl. Tullner, Geschichte, S. 93, Schneider, Sachsen-Anhalt, S. 147.

45 Stenographisches Protokoll der Länderkonferenz vom 11.4. 1947, BAB, DP1 VA Nr. 6958, Bl. $25-27,43 \mathrm{f}$. 
Hilfsarbeiter einzustellen, desgleichen bis zu 50 Kräfte des Bürodienstes“46. Dieser Antrag war angesichts der intransigenten Haltung der Besatzungsmacht in diesem Punkt zum Scheitern verurteilt. Von der DJV konnte das Justizministerium in Halle zudem keine Unterstützung erwarten. Denn Hilde Benjamin hatte bei einem Besuch in Sachsen-Anhalt im April festgestellt, daß für Änderungen der Grundbücher im Zuge der Bodenreform noch 28 Richter, 30 Rechtspfleger, 22 Sekretäre und 24 Schreibkräfte aushilfsweise beschäftigt seien, die nach Befehl Nr. 49 als „belastet“ galten. Aufgrund eines Erlasses des Justizministeriums vom 2. Dezember 1946 war den Gerichten gestattet worden, diese Hilfskräfte auch in der freiwilligen Gerichtsbarkeit einzusetzen. Noch bevor die DJV der SMADRechtsabteilung darüber Bericht erstattete, ließ sie das Justizministerium in Halle wissen, daß der Einsatz der Hilfskräfte in der freiwilligen Gerichtsbarkeit einen Verstoß gegen Befehl Nr. 49 darstelle ${ }^{47}$. Daraufhin wurde der Erlaß vom 2. Dezember zurückgezogen ${ }^{48}$, wenngleich im Zusammenhang mit der Bodenreform „belastete“ Hilfskräfte nachweislich bis Mai 1948 beschäftigt blieben ${ }^{49}$.

Nicht nur in Sachsen-Anhalt, sondern auch in Thüringen war man 1946/47 bestrebt, die strikte Entnazifizierung nach Befehl Nr. 49 zu unterlaufen. Helmut Külz, Direktor des Landesamts für Justiz ${ }^{50}$, erklärte dem Leiter der DJV-Personalabteilung im Juli 1946, „daß man den Befehl Nr. 49 seinem unmittelbaren Wortlaut entsprechend nur anwende auf Mitglieder der NSDAP“. Ehemalige Angehörige der Parteigliederungen konnten somit entgegen der Weisung Schiffers vom 20. September 1945 in Thüringen in ihren Stellungen verbleiben. Dieser wies in einer Rundverfügung nochmals darauf hin, daß Befehl Nr. 49 "dahin auszulegen [sei], daß er sowohl für die Mitglieder der ehemaligen NSDAP selbst gilt wie für die Angehörigen ihrer Gliederungen“. Auch diese Weisung setzte das Weimarer Landesamt für Justiz nur teilweise um, indem „im Einverständnis mit der SMA des Landes Thüringen der Befehl Nr. 49 zwar auch auf Angehörige der SA und SS im vollen Umfange ausgedehnt [wurde], auf Angehörige der sonstigen Gliederungen aber nur insoweit, als diese Mitglieder einen Rang vom Scharführer $\mathrm{ab}$ aufwärts bekleidet haben" 51 . Jedoch auch im Hinblick auf SA-Mitglieder scheint diese Anweisung nicht generell eingehalten worden zu sein, da nach dem Nürnberger Urteil vom 30. September/1. Oktober 1946, das die SA nicht als verbrecherische Organisation einstufte ${ }^{2}$, den Anträgen ehemaliger Mitglieder dieser Parteigliederung auf Einstellung in den Justizdienst in Thüringen bisweilen statt-

46 Justizminister Sachsen-Anhalt an Chef der Verwaltung der SMA Halle, 19. 4. 1947, BAB, DP1 VA Nr. 996, Bl. $105 \mathrm{f}$.

47 Chef der DJV an Provinzialregierung Sachsen-Anhalt/Justizministerium, 26. 4. 1947, Chef der DJV an SMAD-Rechtsabteilung, 29. 4. 1947, ebenda, B1. 116, 115.

48 DJV an SMAD-Rechtsabteilung, Mai 1947, ebenda, Bl. 103.

49 Bericht über die Überprüfung der Justizverwaltung des Landes Sachsen-Anhalt in der Zeit vom 19.-28. 5. 1948, BAB, DP1 VA Nr. 12.

so Das Landesamt für Justiz war der Vorgänger des thüringischen Justizministeriums. Sein Leiter Külz wurde am 4. 12. 1946 Justizminister: vgl. Welsh, Thüringen, S. 184 f., und Weber, Justiz und Diktatur, S. 31.

51 Bericht des Landesamts für Justiz über die Durchführung des Befehls Nr. 49, 16. 10. 1945, BAB, DP1 VA Nr. 996, Bl. 49f.; die Rundverfügung vom 25. 9. 1946 ebenda, Bl. 41.

52 Vgl. IMT Bd. 1, S. $307 \mathrm{ff}$. 
gegeben wurde ${ }^{53}$. Auch 19 ehemalige „Fördernde Mitglieder der SS“ wurden erst aus dem Justizdienst entlassen, als die sowjetische Militärverwaltung in Weimar dies zu Beginn des Jahres 1947 anordnete. In der Hoffnung, daß die thüringische Militärverwaltung damit ihre Kompetenzen überschritten habe, fragte Külz daraufhin bei der DJV nach, ob diese Anordnung im Einklang mit den in der Zentralverwaltung und in Karlshorst vertretenen Grundsätzen stünde ${ }^{54}$. Hilde Benjamin ging jedoch davon aus, "daß der Befehl der thüringischen SMA offensichtlich von Karlshorst veranlaßt" sei ${ }^{55}$; dem Justizminister in Weimar wurde folgerichtig beschieden, daß die DJV der Auffassung der SMA des Landes Thüringen beitrete ${ }^{56}$.

Es gab also durchaus Versuche einzelner Länder, die mit Befehl Nr. 49 angeordnete umfassende Entnazifizierung der Justiz aufzuweichen oder zumindest aufzuschieben. Die DJV hatte sich zwar auf eine konsequente Linie in dieser Angelegenheit festgelegt, verschloß sich anfangs jedoch noch nicht den pragmatischen Argumenten aus einigen Landeshauptstädten, die gegen diese strikte Linie sprachen. Da die SMAD-Rechtsabteilung jedoch konsequent blieb, besaß die DJV in dieser Frage so gut wie keinen Handlungsspielraum und mußte sich im wesentlichen darauf beschränken, die Anordnungen aus Karlshorst weiterzugeben.

Lediglich im Hinblick auf die unter Befehl Nr. 49 fallenden jungen Juristen, die ihre Ausbildung noch nicht abgeschlossen hatten, wurde sie von sich aus tätig. Den Hintergrund bildete die Tatsache, daß einige Länder und Provinzen für die Behandlung der Mitglieder der NSDAP oder ihrer Gliederungen, die noch als Jugendliche beigetreten waren, Sonderregelungen getroffen hatten, die auch auf Angehörige des Justizdienstes übertragen worden waren. Angesichts der strikten Entnazifizierungspolitik der SMAD legten Benjamin - die die Frage aufgeworfen hatte -, Melsheimer und Schiffer am 25. September 1946 in diesem Zusammenhang fest, daß nur ,in ganz vereinzelten Ausnahmefällen, bei wirklich aktiver antifaschistischer Betätigung “ von Befehl Nr. 49 abgewichen werden könne - eine Regelung, die für ehemalige Mitglieder der NSDAP, des SD, der SS, der SA, des NSKK, des NSFK und der NS-Frauenschaft gelten solle. Bei einfachen Mitgliedern der HJ und des NSDStB wollte man diese lockern: „Bei ihnen soll von Fall zu Fall individuell geprüft werden, ob der Bewerber auch objektiv im gegenwärtigen Stadium des Neuaufbaus bereits tragbar ist. Dabei sind insbesondere zu berücksichtigen Familie, Erziehung, Studiengang und auch die Betätigung nach dem Zusammenbruch." Die Entscheidungen über derartige Ausnahmen treffe allein der Chef der DJV57. Mit diesem Beschluß, der am 3. Oktober in Form einer Rundverfügung verbreitet wurde ${ }^{58}$, hatte man vor allem die Jugendlichen im Blick, die bereits vor 1945 ein juristisches Studium aufgenommen oder mit dem

53 So die Feststellung Jakupows auf der Juristenkonferenz vom 11. 4. 1947, BAB, DP1 VA Nr. 6958, Bl. 27.

54 MdJ Thüringen an DJV, 4. 1. 1947, BAB, DP1 VA Nr. 996, Bl. 37.

55 Abteilung II an Amtschef, 21. 1. 1947, ebenda, Bl. 59.

56 DJV an MdJ Thüringen, 25. 1. 1947, ebenda, Bl. 60.

57 Vermerk, 3. 10. 1946, ebenda, Bl. 42.

58 DJV an Landes- und Provinzialverwaltungen/Abteilung Justiz, 3.10. 1946, BAB, DP1 VA Nr. 983, Bl. 5. Die Rundverfügung ging insofern sogar über die Festlegungen vom 25. September hinaus, als die Ausnahmeregelung generell für alle $\mathrm{HJ}$ - und NSDStB-Mitglieder galt. Vgl. auch Pohl, Justiz in Brandenburg (Manuskript), S. 23. 
Referendariat begonnen hatten. Für die Zulassung zur Referendar- und Assessorprüfung sollten daher nicht die Bestimmungen der jeweiligen Prüfungsordnung ${ }^{59}$, sondern die der Verfügung vom 3. Oktober gelten ${ }^{60}$. Ohne eine Lockerung der strikten Entnazifizierungsvorschriften von Befehl Nr. 49 hätte man auf das Reservoir an jungen, bereits juristisch vorgebildeten Nachwuchskräften wohl kaum zurückgreifen können.

Eine neue Situation für die „belasteten“ jüngeren Justizbediensteten schien mit den sogenannten "Jugendamnestien" von Februar und März 1947 zu entstehen. Die Landtage verabschiedeten damals Gesetze, denen zufolge auf nach dem 1. Januar 1919 geborene Personen die einschränkenden Bestimmungen von Gesetzen, die ehemalige Mitglieder der NSDAP oder deren Gliederungen betrafen, keine Anwendung mehr fanden ${ }^{61}$. Den Hintergrund bildete die dritte Entnazifizierungswelle nach Kontrollratsdirektive Nr. 24, die im Herbst 1946 in der SBZ anlief. Bemerkenswert ist, daß die "Jugendamnestien“ in ihren Vorschriften identisch sind, was möglicherweise auf eine zentrale Steuerung zurückging ${ }^{62}$. Noch bevor der Landtag in Dresden das Gesetz verabschiedet hatte, fragte am 31. Januar das sächsische Justizministerium bei der DJV an, ob dadurch die bisherigen, auf Befehl Nr. 49 beruhenden Regelungen beeinträchtigt würden ${ }^{63}$. Die entschied, daß sich aufgrund der in Aussicht stehenden Jugendgesetze nichts an der bisherigen Entnazifizierungspraxis im Hinblick auf die jungen Juristen ändern werde, da der Justiz eine Sonderstellung im öffentlichen Leben zukomme ${ }^{64}$.

Denn die DJV wollte - wie eine Auseinandersetzung mit Thüringen zeigt - sich die Entscheidung über die Ausnahmen selbst vorbehalten. Dort beließ man ehemalige HJ- und NSDStB-Mitglieder im Justizdienst, wenn sie von den örtlichen gemäß Kontrollratsdirektive Nr. 24 gebildeten Entnazifizierungskommissionen als tragbar befunden worden waren. Demgegenüber betonte Melsheimer in Vertretung von Schiffer, „daß die Zulassung ehemaliger Mitglieder der HJ und des Studentenbundes [...] nach wie vor grundsätzlich durch die Deutsche Justizverwaltung erfolgt". Lediglich bei den Angehörigen des einfachen und mittleren Dienstes erklärte er die Überprüfung der Kommissionen für ausreichend ${ }^{65}$. Den-

59 Referendarprüfungsordnung vom 16.12. 1946, BAB, DP1 VA Nr. 1000, ( $\ 1 \mathrm{a})$; Assessorprüfungsordnung vom 16. 1. 1946, BAB, DP1 VA Nr. 7854 ( $\$ 3$, Abs.6).

$60 \mathrm{Vgl}$. DJV an Provinzialverwaltung Sachsen/Abteilung Justiz, 13. 12. 1946 (auch an die anderen Justizabteilungen geschickt), BAB, DP1 VA Nr. 983, Bl. 4; Äußerung Benjamins in: Vermerk über die Dienstbesprechung vom 22. 1. 1947, BAB, DP1 VA Nr. 7354.

6) Gesetz zur Durchführung der Direktive 24 des Alliierten Kontrollrates für Jugendliche, die nach dem 1. Januar 1919 geboren wurden, 17. 2. 1947, in: Regierungsblatt für das Land Thüringen I, 1947, S. 6 f.; Gesetz zur Entnazifizierung von Personen, die seit dem 1. Januar 1919 geboren wurden, 19. 3. 1947, in: Gesetzblatt der Provinz Sachsen-Anhalt I, 1947, S. 60; Gesetz über die Behandlung der jugendlichen ehemaligen Mitglieder der NSDAP und ihrer Gliederungen, 27.3. 1947, in: Gesetze/Befehle, Verordnungen und Bekanntmachungen durch die Landesverwaltung Sachsen I, 1947, S. 135.

62 Eine Anregung von sowjetischer Seite erscheint unwahrscheinlich, da die SMAD die von den Landtagen verabschiedeten Gesetze aufhob: vgl. Wille, Entnazifizierung, S. 149.

${ }^{63}$ Die Anfrage lag nicht vor; für Datum und Inhalt der Anfrage siehe Chef der DJV an Landesregierung Sachsen/Justizministerium, 18. 2. 1947, BAB, DP1 VA Nr. 996, Bl. 69.

64 Siehe Chef der DJV an Landesregierung Sachsen/MdJ, 18. 2. 1947, ebenda, und ähnlich formulierte Schreiben an die Justizministerien der übrigen Länder und Provinzen vom selben Datum, BAB, DP1 VA Nr. 7355.

65 Chef der DJV an Landesregierung Thüringen/MdJ, 21. 3. 1947, ebenda. 
noch wartete die DJV im Frühjahr 1947 vergebens darauf, daß das thüringische Justizministerium ihr die Fälle ehemaliger HJ- und NSDStB-Mitglieder im Justizdienst zur Entscheidung vorlegte ${ }^{66}$. Wie Otto Hartwig auf einer Dienstreise nach Thüringen feststellte, lag dies daran, daß das Weimarer Justizministerium die Rundverfügung vom 3. Oktober 1946 erst am 21. April 1947 an das für die Referendare zuständige Oberlandesgericht weitergeleitet hatte ${ }^{67}$ - ein Hinweis darauf, daß Anweisungen aus Berlin von der thüringischen Justizverwaltung nicht allzu ernst genommen wurden.

Hinzu kam, daß Ministerpräsident Paul sich nicht mit der Entscheidung der DJV abgefunden hatte, derzufolge die sogenannte "Jugendamnestie" nicht für das Justizpersonal gelten solle. Über Oberlandesgerichtspräsident Barth wandte er sich daher an Schiffer mit der Bitte, in diesem Sinne in Berlin-Karlshorst vorzusprechen ${ }^{68}$. Dieser ließ Barth aber am 31. Mai 1947 mitteilen, er verspreche sich "nach Lage der Sache von weiteren Schritten in der Angelegenheit keinen Erfolg“". Zwar bedauerte er, daß durch diese Verfahrensweise „manche sachlich voll geeignete Kräfte der Justiz ferngehalten“" würden, es sei jedoch „keine ausreichende Gewähr dafür gegeben, daß diese Kräfte vom Geiste der Demokratie [...] erfüllt" seien ${ }^{69}$. Die DJV hatte zudem bereits drei Tage zuvor verfügt, daß trotz der „Jugendamnestie" die Regelung vom 3. Oktober weiterhin gültig sei, und daher vor Zulassung zur Referendarprüfung der Kandidat im Hinblick auf seine frühere $\mathrm{Zu}$ gehörigkeit zur HJ oder dem NSDStB überprüft und gegebenenfalls eine Entscheidung des Chefs der DJV herbeigeführt werden müsse ${ }^{70}$. Auch dagegen erhob Külz Einspruch, indem er darauf verwies, daß die Referendarprüfung nicht ausschließlich eine Eingangsprüfung für den Justizdienst, sondern auch „die Voraussetzung für die Laufbahn der künftigen Wirtschafts- und Verwaltungsjuristen, für den akademischen Juristennachwuchs und für andere juristische Berufe" sei. Wenn man Befehl Nr. 49 auf die erste juristische Staatsprüfung ausdehne, so fuhr er fort, könne diese wegen der hohen Anzahl der HJ-Mitglieder unter den Studenten „nur noch in Ausnahmefällen abgenommen werden“, und die Studenten würden zur Ablegung dieser Prüfung in eine andere Besatzungszone abwandern. Aufgrund dieser Bedenken habe er dem juristischen Prüfungsamt und der Universität Jena zwar von dem Erlaß vom 28. Mai Kenntnis gegeben, „aber angeordnet, von seiner Beachtung vorläufig abzusehen"71. Mit dieser Argumentation stieß Külz bei Melsheimer, der als Kommunist für eine strikte Entnazifizierung der Justiz eintrat ${ }^{72}$, auf schroffe Ablehnung: Das Referendarexamen sei „eine Justizeingangs- [und] keine Universitätsabschlußprüfung“. Demgegenüber sah Winkelmann keine Gefahren für die Justiz bei der Examenszulassung von unter Befehl

$66 \mathrm{Vgl}$. DJV an Landesregierung Thüringen/MdJ, 29. 4. 1947, BAB, DP1 VA Nr. 996, B1. 95.

67 Vermerk Hartwigs für Benjamin, 22. 5. 1947, ebenda, Bl. 117.

68 Vermerk Hartwigs für Schiffer, 22. 5. 1947, BAB, DP1 VA Nr. 983, Bl. 14.

69 Hartwig an Barth, 31. 5. 1947, ebenda, Bl. 15; vgl. Weber, Justiz und Diktatur, S. 42.

70 DJV an Landes- und Provinzialregierungen/Justizministerium, 28. 5. 1947, ebenda, Bl. 29.

71 Justizministerium Thüringen an DJV, 18. 7. 1947, BAB, DP1 VA Nr. 996, Bl. $145 \mathrm{f}$.

72 Vgl. Melsheimers Vorlage für Ulbricht zur Reform des Justizwesens (Anfang 1946), SAPMO, NY 4105/3, Bl. 18-20, hier 19. 
Nr. 49 fallenden Personen, sondern plädierte dafür, diese lediglich vom Referendardienst auszuschließen ${ }^{73}$.

Auffällig war, daß - im Unterschied zur allgemeinen Praxis bei Befehl Nr. 49 die DJV ihre Linie bezüglich der Entnazifizierung der jungen Juristen selbständig festlegte und Karlshorst sich hier nicht einmischte. Die Zentralverwaltung verfolgte freilich keinen von der SMAD-Rechtsabteilung abweichenden Kurs. Dies verdeutlichte Jakupow, der auf der Länderkonferenz am 11. April 1947 verkündete, daß die unter die "Jugendamnestie" fallenden HJ- und SA-Mitglieder keineswegs zum Dienst in den Justizbehörden zugelassen werden dürften ${ }^{74}$. Trotz der Rückendeckung für die Politik der DJV wandte sich Schiffer angesichts des hartnäckigen Widerspruchs aus Thüringen am 30. Juni 1947 an Nikolajew mit der Bitte um Klärung der Angelegenheit ${ }^{75}$. Gemäß der am 7. August übermittelten Anordnung der SMAD-Rechtsabteilung war den nominellen Mitgliedern der NSDAP oder ihrer Gliederungen nach bestandenem Examen die Beschäftigung in der Justiz zu ermöglichen, jedoch nur als Rechtsanwalt, Notar oder technischer Angestellter; zu Richtern, Staatsanwälten oder auf sonstige Führungsposten durften sie „in keinem Fall“ ernannt werden ${ }^{76}$. Damit war zwar der Kreis der von der Regelung Profitierenden über die HJ- und NSDStB-Mitglieder hinaus erweitert worden; da keine der unter Befehl Nr. 49 fallenden Personen jedoch zum Richteramt zugelassen werden durfte, bedeutete die Weisung de facto eine Verschärfung der von der DJV sanktionierten Praxis. Was die SMAD zu dieser Kurskorrektur veranlaßte, läßt sich nicht eindeutig bestimmen; möglicherweise stand sie im Zusammenhang mit der allgemeinen Verschärfung der Entnazifizierung im Zuge der Umsetzung von Kontrollratsdirektive Nr. 24. Im Hinblick auf die Zulassung „belasteter“ junger Juristen legte Schiffer zwei Tage später fest, „daß es bei der bisherigen Regelung verbleibt, d.h. daß letzten Endes die DJV entscheidet" 77 . In einer weiteren DJV-internen Beratung am 20. August wurde als letzte Frage in diesem Zusammenhang geklärt, daß eine Überprüfung durch die DJV nicht für die Zulassung von politisch „Belasteten“ zur ersten juristischen Staatsprüfung, sehr wohl aber für deren Ernennung zum Referendar erforderlich sei ${ }^{78}$. Nachdem die SMAD-Rechtsabteilung dem zugestimmt hatte, erging am 27. September 1947 ein entsprechender Erlaß an die Justizministerien der Länder, womit die Rundverfügung vom 28. Mai aufgehoben und letztlich den vom thüringischen Justizminister ausgesprochenen Bedenken Rechnung getragen wurde ${ }^{79}$.

73 Vermerk Winkelmanns, 31. 7. 1947, BAB, DP1 VA Nr. 996, Bl. 147.

74 Stenographisches Protokoll der Länderkonferenz vom 11. 4. 1947, BAB, DP1 VA Nr. 6958, Bl. 27.

75 Vermerk über die Unterredung Schiffers und Nikolajews am 30.6. 1947, BAB, DP1 VA Nr. 11, Bl. 118.

76 Aktenvermerk über die Besprechung Jakupows mit Melsheimer am 7. 8. 1947, ebenda, Bl. 122. Eine entsprechende, von Karassjow und Malkow unterzeichnete Direktive ging im August 1947 an die Länder-SMAs: vgl. Nikitin, Sowjetische Militäradministration und die Justiz (Manuskript), S. 11.

77 Vermerk Winkelmann über eine Besprechung am 9. 8. 1947, BAB, DP1 VA Nr. 996, Bl. 151.

78 Vermerk über eine Besprechung von Hartwig, Melsheimer, Benjamin und Schiffer am 20. 8. 1947, BAB, DP1 VA Nr. 983, Bl. 42.

79 DJV an SMAD-Rechtsabteilung, 25. 8. 1947, BAB, DP1 VA Nr. 996, Bl. 157; SMAD-Rechtsabteilung an Chef der DJV, 12. 9. 1947, ebenda, Bl. 162; Chef der DJV an Landesregierungen/Justizministerium, 27. 9. 1947, BAB, DP1 VA Nr. 983, Bl. 45. 
Während bei der allgemeinen Durchführung von SMAD-Befehl Nr. 49 die DJV so gut wie keine Eigenständigkeit zeigte und ihr durch die ständigen Eingriffe der Rechtsabteilung aus Karlshorst die Hände gebunden waren, versuchte sie bei dem Spezialfall der Anwendung des Befehls auf junge Juristen eine eigene Linie zu entwickeln ${ }^{80}$. Angesichts des ungeheuren Personalmangels wollten die führenden Mitarbeiter der DJV die jungen, lediglich durch Mitgliedschaft in der $\mathrm{HJ}$ oder dem NSDStB „belasteten“, aber noch „formbaren“ Juristen ${ }^{81}$ nicht gänzlich verlieren und waren daher bereit, von der sonst rigiden Entnazifizierungspraxis abzuweichen. Die Gewährung von Ausnahmen auf diesem sensiblen Gebiet behielt sich die DJV freilich selber vor. Wollten Schiffer und die ihm zuneigenden Mitarbeiter auf diese Weise gewährleisten, daß keine Kräfte in die Justiz übernommen wurden, die ihre Reformvorhaben behinderten, sahen Benjamin und Melsheimer darin eine Möglichkeit, kommunistische „Kaderpolitik“ $z u$ betreiben. Als gegen das Vorgehen der DJV Widerspruch aus den Ländern kam, wandte sich diese nach ersten Bemühungen, den eigenen Kurs durchzusetzen, aufgrund von Bedenken, eine "falsche" Entscheidung zu treffen, an die SMAD, die letztlich auch in diesem Bereich ein strikteres Vorgehen als die deutsche Zentralverwaltung anordnete. Auch wenn die Militärverwaltung auf eigene Regelungen zunächst verzichtete und die DJV gewähren ließ, ergab sich aus dem Herrschaftsgefüge in der SBZ, daß bei Konflikten zwischen der Zentralverwaltung und den regionalen Justizministerien die Besatzungsmacht als Schlichter herangezogen und deren Stellung somit gefestigt wurde.

Bei allen Schwierigkeiten und Problemen wurde die Entnazifizierung des Justizpersonals in der SBZ äußerst gründlich durchgeführt, wenngleich Unterschiede zwischen den einzelnen Ländern bestanden 82 . Bereits die DJV hatte Probleme, die Anzahl der vor Erlaß von Befehl Nr. 49 Entlassenen zu ermitteln: Während in Mecklenburg angeblich keiner der unter dem Nationalsozialismus tätigen Richter und Staatsanwälte weiter beschäftigt wurde ${ }^{83}$ und aus der Provinz Sachsen keine Zahlen vorlagen, schieden in Thüringen, Sachsen und Brandenburg insgesamt 502 Richter und 66 Staatsanwälte aufgrund von Entnazifizierungsmaßnahmen aus dem Justizdienst aus ${ }^{84}$. Unmittelbar nach Erlaß von Befehl Nr. 49, im Herbst 1945 folgte dann die wohl radikalste Säuberungswelle im Justizwesen: Bis

80 Dabei handelte es sich freilich um ein eher marginales Problem: Von den insgesamt 67 bis September 1947 vorliegenden Fällen waren 24 noch nicht entschieden; 13 Referendare wurden zugelassen, 30 abgelehnt: Maßnahmen zur Entfernung der nazistischen Elemente aus der Justiz, 19.9. 1947, BAB, DP1 VA Nr. 821, BI. 89.

81 Vgl. in diesem Zusammenhang jedoch den Einwand von Hilde Benjamin, „daß Richterkräfte, die die jungen Juristen weltanschaulich richtig beeinflussen, nicht in genügendem Maße vorhanden seien": Vermerk über die Besprechung vom 16. 10. 1946, BAB, DP1 VA Nr. 7354.

82 Zum Ausmaß der "Säuberungen“ in der Justiz der SBZ siehe u.a. Meinicke, Entnazifizierung, S. 141-147; Wille, Entnazifizierung, S. 76-82; Lorenz, Zentralverwaltung, S. 157 f.; Amos, Justizverwaltung, S. 138-149. Da in diesen Arbeiten die Zahlen differenziert nach Ländern vorliegen, wird hier auf eine detaillierte Darstellung verzichtet.

83 Vgl. Benjamin u. a., Zur Geschichte der Rechtspflege 1945-1949, S. 54. Nach Erlaß von Befeh] Nr. 49 wurde dies von der Schweriner Landesverwaltung am 25. 9. 1945 bekräftigt: vgl. Anders, Demokratisierung, S. 38. Dennoch wurden aufgrund des Befehls noch 18 Richter und 3 Staatsanwälte entlassen: siehe Lorenz, Zentralverwaltung, S. 158. Vgl. dazu auch Bartusel, Politisierung, S. $27 \mathrm{f}$.

${ }_{84}$ Übersicht, 27. 6. 1946, BAB, DP1 VA Nr. 996, Bl. 31; vgl. Lorenz, Zentralverwaltung, S. 158. 
Ende Juni 1946 wurden in allen Ländern und Provinzen 579 Richter und 78 Staatsanwälte entlassen ${ }^{85}$. Bis zum 23. August 1947 wurden weitere 228 Richter und 30 Staatsanwälte entfernt, so daß insgesamt 807 Richter und 108 Staatsanwälte als Ergebnis der Säuberungen nach Befehl Nr. 49 aus dem Dienst ausschieden ${ }^{86}$. Hinzu kamen 6868 weitere Justizbedienstete ${ }^{87}$; nach dem Stand vom 15. September 1947 waren daher nur noch acht Richter, 14 Staats- und Amtsanwälte sowie 1350 sonstige Personen im Justizdienst beschäftigt, die der NSDAP oder einer ihrer Gliederungen angehört hatten ${ }^{88}$.

Die Entnazifizierung der Justiz in der SBZ erfolgte seit Dezember 1946 auch aufgrund von Kontrollratsdirektive Nr. 24 vom 12. Januar 194689. Im Unterschied zu Befehl Nr. 49 galt die Direktive der Mehrheit der ehemaligen juristischen, wirtschaftlichen und administrativen Elite Deutschlands, die aufgrund ihrer beruflichen Stellung oder ihrer sozialen und kulturellen Herkunft der Gegnerschaft zur Demokratie verdächtigt wurde ${ }^{90}$. Über Verbleib oder Entlassung der einzelnen Verdächtigen entschieden Entnazifizierungskommissionen, die ab Herbst 1946 auf Landesebene, in den Städten, Kreisen und in bestimmten öffentlichen Einrichtungen (wie im Postwesen und bei der Reichsbahn) gebildet wurden ${ }^{91}$. Die DJV besaß dabei keine Kompetenzen ${ }^{92}$ und beschränkte sich darauf, Berichte über deren Durchführung bezüglich des Justizdienstes anzufordern'33. Aufgrund der strikten Durchführung von Befehl Nr. 49 wurden jedoch bis September 1947 aufgrund der Direktive Nr. 24 nur 68 Richter, 10 Staatsanwälte und 268 sonstige Justizbedienstete in Mecklenburg, Brandenburg, Thüringen und Sachsen und in Sachsen-Anhalt insgesamt sechs Justizangehörige entlassen ${ }^{94}$.

Auch die Rechtsanwaltschaft wurde entnazifiziert, zunächst auf der Grundlage von Landesgesetzen und -verordnungen ${ }^{95}$. Da die DJV im Hinblick auf Zulassung und Tätigkeit der Rechtsanwälte und Notare für sich ein Kontrollrecht beanspruchte, entwarf sie für beide Berufsgruppen Zulassungsverordnungen, die vom

85 Übersicht, 27. 6. 1946, BAB, DP1 VA Nr. 996, Bl. 31.

86 Maßnahmen zur Entfernung der nazistischen Elemente aus der Justiz, 19. 9. 1947, BAB, DP1 VA Nr. 821, Bl. 88. Zahlen auch in Lorenz, Zentralverwaltung, S. 158. Einem Bericht der DJV an die SMAD-Rechtsabteilung vom 6.9. 1948 zufolge wurden insgesamt 811 Richter und 112 Staatsanwälte aufgrund von Befehl Nr. 49 entlassen, BAB, DP1 VA Nr. 821, Bl. 185; vgl. auch Amos, Justizverwaltung, S. 142.

87 DJV an SMAD-Rechtsabteilung, 6. 9. 1945, BAB, DP1 VA Nr. 821, Bl. 185.

${ }_{88}$ Maßnahmen zur Entfernung der nazistischen Elemente aus der Justiz, 19. 9. 1947, ebenda, Bl. 88.

89 Gedruckt in: Amtsblatt des Kontrollrats Nr. 5, 31. 3. 1946, S. 98-115.

90 Vgl. Absätze 10-12 der Direktive und van Melis, Entnazifizierung in Mecklenburg-Vorpommern, S. 192.

9s Vgl. dazu u. a. Wille, Entnazifizierung, S. $139 \mathrm{f}$.

92 Benjamin sah gleichwohl den Vorteil von Kontrollratsdirektive Nr. 24, die auch Kräfte erfasse, "die nicht einmal unter den Befehl Nr. 49 fielen" (Vermerk über die Besprechung vom 22. 1. 1947, BAB, DP1 VA Nr. 7354).

93 DJV an Provinzial- und Landesregierungen/Justizministerium, 24.9. 1947, BAB, DP1 VA Nr. 823, Bl. 14.

94 Maßnahmen zur Entfernung der nazistischen Elemente aus der Justiz, 19. 9. 1947, BAB, DP1 VA Nr. 821, B1. 89. Abgeschlossen wurde die Überprüfung der Justiz jedoch nur in Sachsen. Vgl. auch Lorenz, Zentralverwaltung, S. 158. Im Bericht an die SMAD-Rechtsabteilung vom 6.9. 1948, BAB, DP1 VA Nr. 821, Bl. 185, werden 70 Richter, 7 Staatsanwälte und 267 sonstige Entlassene nach Direktive Nr. 24 genannt.

95 Vgl. Lorenz, Rechtsanwaltschaft in der SBZ und der DDR, S. 524. 
Obersten Chef der SMAD bestätigt wurden ${ }^{96}$. Schiffer und seine Abteilungsleiter hatten dabei von vornherein eine weniger rigorose Entnazifizierungspraxis als in den anderen Justizbehörden vorgesehen, da die Rechtsanwaltschaft „keine mit Zwangsgewalt versehene Hoheitsstelle" sei"7. Die Provisorische Zulassungsordnung für die Rechtsanwaltschaft schloß daher lediglich diejenigen Mitglieder der NSDAP aus, „die sich mehr als nur nominell an ihrer Tätigkeit beteiligt haben“, sowie die Personen, „die bei der Strafpraxis des Hitlerregimes mitgewirkt haben“. Als nominelle Mitglieder galten nach den internen Durchführungsbestimmungen diejenigen, die sich über die Zahlung ihrer Mitgliedsbeiträge hinaus nicht in der Partei engagiert hatten. Auch sie waren freilich nur zuzulassen, "wenn sie den Nachweis ihrer antifaschistischen Einstellung erbringen und die Gewähr dafür bieten, daß sie sich als Rechtsanwälte im antifaschistisch-demokratischen Sinne betätigen werden"98. Da mit der Provisorischen Zulassungsordnung vom 18. Juni 1946 die bis dahin gültigen Zulassungen außer Kraft gesetzt wurden und die Rechtsanwälte bis zum 1. Oktober 1946 ihre Neuzulassung beantragen mußten, setzte sich die DJV - wenn auch nicht ohne Auseinandersetzungen - gegenüber den unterschiedlichen regionalen Regelungen durch ${ }^{99}$. Zum 1. April 1947 waren unter 996 in der SBZ zugelassenen Rechtsanwälten 283 ehemalige Mitglieder der NSDAP oder ihrer Gliederungen ${ }^{100}$. Die SMAD-Rechtsabteilung hätte gern eine restriktivere Zulassungspraxis gesehen ${ }^{101}$, so daß Jakupow im April 1947 die große Anzahl ehemaliger Nationalsozialisten beanstandete, die noch in der Anwaltschaft, insbesondere in Thüringen und Sachsen, tätig seien ${ }^{102}$. Nach einer daraufhin von der DJV in Sachsen durchgeführten Überprüfung konnte die Anzahl der „belasteten“ Anwälte von 60 auf zwei im September 1947 verringert werden. $\mathrm{Da}$ sich in anderen Ländern, wie beispielsweise in Thüringen, deren Anzahl sowohl absolut als auch prozentual erhöhte, war bis zum September 1947 nur ein verhältnismäßig geringer Rückgang zu verzeichnen: Einer Aufstellung der DJV zufolge befanden sich damals noch 256 ehemalige Mitglieder der NSDAP oder ihrer Gliederungen unter den 1008 Anwälten - das entsprach 25,4 Prozent gegenüber 28,4 Prozent im April103. Sowohl die absolute Anzahl als auch der Prozent-

96 Provisorische Zulassungsordnung für die Rechtsanwaltschaft in der Sowjetischen Besatzungszone Deutschlands, 18. 6. 1946; Verordnung über die Bestellung zum Notar in der Sowjetischen Besatzungszone Deutschlands, 26. 9. 1946, BAB, DP1 VA Nr. 879, Bl. 16f., 11 f. Die Provisorische Zulassungsordnung für die Rechtsanwaltschaft ist u.a. gedruckt in: Amtsblatt für die Landesregierung Mecklenburg-Vorpommern, Nr. 11, 22. 10. 1946, S. 123. Vgl. dazu Lorenz, Rechtsanwaltschaft in der DDR, S. 22-24.

97 Vermerk Schiffers, 7. 2. 1946, BAB, DP1 VA Nr. 6463, Bl. $21 \mathrm{f}$.

98 Durchführungsbestimmungen zu der Provisorischen Zulassungsordnung für die Rechtsanwaltschaft, o.D., BAB, DP1 VA Nr. 879, Bl. 14 f.; teilweise zit. bei Lorenz, Zentralverwaltung, S. $159 \mathrm{f}$. Auch die Verordnung über die Bestellung zum Notar und die dazugehörige Durchführungsbestimmung enthalten fast gleichlautende Regelungen.

99 Vgl. Punkt VI der Durchführungsbestimmungen zu der Provisorischen Zulassungsordnung für die Rechtsanwaltschaft, o.D., BAB, DP1 VA Nr. 879, Bl. 15; Maßnahmen zur Entnazifizierung der Justiz, BAB, DP1 VA Nr. 821, Bl. 89 f.; Lorenz, Zentralverwaltung, S. 160; ders., Rechtsanwaltschaft in der SBZ und der DDR, $523 \mathrm{f}$.

100 Ders., Zentralverwaltung, S. 160.

101 Vgl. dazu Benjamin in der Dienstbesprechung vom 22. 1. 1947, BAB, DP1 VA Nr. 7354.

102 Stenographisches Protokoll der Länderkonferenz vom 11.4. 1947, BAB, DP1 VA Nr. 6958, Bl. $25 \mathrm{f}$.

${ }_{103}$ Vgl. Maßnahmen zur Entnazifizierung der Justiz, BAB, DP1 VA Nr. 821, Bl. 90; die Zahlen vom 
satz der „belasteten“ Anwälte ging in den nächsten Jahren indes nicht weiter zurück, sondern erhöhte sich, da viele aus den Justizbehörden ausscheidende Richter oder Staatsanwälte als Anwälte zugelassen wurden.

Für die Sowjetunion stand bereits 1945 fest, daß im Justizwesen kein nationalsozialistischer Richter oder Staatsanwalt verbleiben sollte. Die Entnazifizierung war somit eines der wenigen Vorhaben, das von der SMAD konsequent vorangetrieben wurde. Entscheidend war dabei die übergeordnete politische Zielsetzung, die traditionellen deutschen Führungsschichten aus ihren Ämtern zu verdrängen, um ein für alle Mal die Wiedererrichtung eines antisowjetisch ausgerichteten Systems in Deutschland zu verhindern. Dies erklärt die Rigorosität der sowjetischen Vorgehensweise, an der weder die DJV noch die Landesjustizverwaltungen etwas ändern konnten. Die DJV unter Schiffer verfolgte zwar - wenn auch aus anderen Motiven - in dieser Frage einen ähnlichen Kurs, verschloß sich aber nicht von vornherein den Argumenten der Landesjustizverwaltungen, die aus pragmatischen Gründen nicht so radikal vorgehen wollten. Lediglich bei der Entnazifizierung der jüngeren, nur durch eine HJ- bzw. NSDStB-Mitgliedschaft „belasteten“ Juristen entwickelte die DJV eine moderatere Linie, die die großzügige Gewährung von Ausnahmen im Einzelfall vorsah. Als sie jedoch bei Konflikten mit den Ländern die SMAD-Rechtsabteilung um eine Entscheidung bat, wurde auch in diesen Fällen ein strikteres Vorgehen verordnet. Die sowjetische Besatzungsmacht nahm die eingeschränkte Funktionsfähigkeit der deutschen Justiz durchaus in Kauf und betrieb damit eine Politik, die der der Westmächte völlig entgegengesetzt war. Das Vorgehen der britischen Besatzungsmacht richtete sich nicht primär gegen die ehemaligen Eliten in der Justiz, sondern gegen die stark nationalsozialistisch „belasteten“ Justizjuristen. Gleichzeitig war ihr an der Aufrechterhaltung der Funktionsfähigkeit der deutschen Justiz gelegen, so daß sie bei den ehemaligen Nationalsozialisten in der Justiz zwischen den stärker und den weniger „belasteten“ zu differenzieren versuchte ${ }^{104}$. Schon Ende Oktober 1945 akzeptierte die britische Besatzungsmacht aufgrund von Vorhaltungen des Präsidenten des Oberlandesgerichts Celle die sogenannte „Huckepack“-Regel, die besagte, daß für jeden „unbelasteten“ Justizjuristen ein ehemaliger Pg eingestellt werden durfte. Damit waren freilich die Dämme gebrochen, überall wurde versucht, diese Regel zu unterlaufen, bis sie am 26. Juni 1946 auch offiziell außer Kraft gesetzt wurde. Damit konnten die „Belasteten“ in Scharen auf ihre alten Posten zurückkehren, und Anfang 1948 waren 30 Prozent der leitenden und 80 bis 90 Prozent der anderen Stellungen im Justizwesen der britischen Zone wieder mit ehemaligen Nationalsozialisten besetzt ${ }^{105}$. Dazu hatte nicht nur der Versuch, zwischen den stärker und weniger „belasteten“ Juristen zu unterscheiden, sondern auch die Tatsache beigetragen, daß die britischen Behörden den Deutschen sehr weitgehende Mitspracherechte zubilligten. Darin freilich unterschieden sich die Westmächte elementar von der Sowjetunion.

September nach einer am 20. 9. 1947 an die SMAD-Rechtsabteilung übermittelten Aufstellung der bei den Justizbehörden tätigen Personen, ebenda, Bl. 83 .

104 Vgl. Rüping, Staatsanwälte und Parteigenossen, S. 28; Wenzlau, Wiederaufbau, S. 129.

105 Ebenda, S. 130-136; Rüping, Staatsanwälte und Parteigenossen, S. 30 f., 86. 